\title{
Planificación territorial y distribución de los beneficios de la parroquia Chumblín Cantón San Fernando
}

Territorial planning and distribution of benefits in the Chumblin parish, San Fernando canton.

Mayra Alexandra Pazato Dutan. ${ }^{1}$, Dorian Damián Flores Aguilera. ${ }^{2} \&$ Yonimiler Castillo Ortega. ${ }^{3}$

\section{Abstract.

Introduction. Territorial planning is very important because its main objective is to analyze the existing problems generated by the actions carried out daily by human beings, which are reflected in inequalities in various scenarios such as: environmental, economic, cultural and/or social problems of the various inhabitants; knowing the existing problems in each territory, it seeks to provide solutions in a given time so that when each of the projects established within the territorial planning materializes, a fair and equitable development for the population is achieved as a result. Objective. Design strategies for a land management plan that takes into account equity in the distribution of benefits in the parish of Chumblín, canton San Fernando. Methodology. The theoretical foundation and research methodology, with a qualitative and quantitative technique by using interviews that were directed to the presidents and social actors of the precincts of the parish. Results. Once the research part was concluded, we proceeded to carry out the procedure and results of the data collection on the aforementioned case study, the verification of the

\footnotetext{
${ }^{1}$ Universidad Católica de Cuenca, Posgrado, Maestría en Desarrollo Local Mención en Ordenamiento Territorial, Cuenca, Ecuador, email mayra.pazato@ucacue.edu.ec, https://orcid.org/0000-0002-6169-526X 2 Universidad Católica de Cuenca, Carrera de economía, Cuenca, Ecuador, email dorian.flores@ucacue.edu.ec, https://orcid.org/0000-0003-0762-8928

${ }^{3}$ Universidad Católica de Cuenca, Posgrado, Maestría en Desarrollo Local Mención en Ordenamiento Territorial, Cuenca, Ecuador, email ycastilloo@ucacue.edu.ec, https://orcid.org/0000-0002-7710-5199
} 
hypothesis was carried out, which allowed us to confirm that the budget of the parish GAD in the period of 2009-2019, has not been distributed equitably, these results have allowed us to generate strategies for the development and improvement of the planning processes that are developed within the administration of the parish GAD that should be governed by the planning laws and thus make a fair distribution of the benefits within the territory. Conclusion. The population of this parish does not participate in the planning of the territory, causing that the distribution of economic resources is not carried out in an equitable way.

Keywords: Planning, Land use planning, Benefits equity, Development

\section{Resumen}

Introducción. La planificación territorial es muy importante ya que tiene como objetivo principal analizar la problemática existente generada por las acciones que realiza a diario el ser humano reflejándose en desigualdades en varios escenarios como son: ambientales, económicas, culturales y/o sociales de los diversos habitantes; conociendo la problemática existente en cada territorio se busca dar solución en un tiempo determinado de manera que al materializarse cada uno de los proyectos que se establece dentro de la planificación territorial se logre como resultado un desarrollo justo y equitativo para la población. Objeto. Diseñar estrategias para un plan de ordenamiento territorial que tenga en cuenta la equidad en la distribución de los beneficios de la parroquia de Chumblín, cantón San Fernando. Metodología. Se estableció la fundamentación teórica y la metodología de investigación, con una técnica cualitativa y cuantitativa utilizando las entrevistas que fueron dirigidas a los presidentes y actores sociales de los recintos de la parroquia. Resultados. Concluido la parte investigativa se procedió a realizar el procedimiento y resultados de la recolección de datos sobre el mencionado caso de estudio, se realiza la comprobación de la hipótesis planteada, misma que nos permitió confirmar que el presupuesto del GAD Parroquial desde el periodo 2009-2019, no se ha distribuido de manera equitativa estos resultados han permitido generar estrategias para el desarrollo y mejoramiento de los procesos de planificación que se desarrollan dentro de la administración del GAD Parroquial que deberán regirse las leyes de planificación y de esta manera realizar un distribución justa de los beneficios dentro del territorio. Conclusión. La población de la parroquia no es participe de la planificación del territorio, provocando que no se realice una distribución de los recursos económicos de forma equitativa.

Palabras claves: Planificación, Ordenamiento Territorial, Equidad de beneficios, Desarrollo. 


\section{Introducción.}

\section{Aspectos teóricos de la planificación del ordenamiento territorial, sus componentes y estructura}

Para tener conocimiento sobre la planificación del ordenamiento territorial debemos tener conocimiento el significado tiene la palabra territorio. Según Geiger (1996) y Goueset (1999), es un espacio físico que tiene relación de poder o posesión por parte de una persona o grupos sociales con ideas de generar desarrollo manteniendo una dimensión culturalista. Sin embargo, Claval (1966) involucra las prácticas y experiencias que permiten la apropiación del territorio y construcción de la identidad del grupo de personas que lo habitan. También considera "Siendo también de pertenencia del territorio a través de un proceso de identificación y de representación, bien sea colectivo o individual, que muchas veces desconoce las fronteras políticas o administrativas".

Las definiciones anteriormente expresadas por los distintos escritores coinciden que el territorio es un espacio físico delimitado, llegando a ser espacios de asentamientos de la población. Cabe mencionar que dentro de estos territorios se generan potestades políticas y sus competencias administrativas, con una finalidad de dividir el territorio en aspectos históricos, geográficos y culturales, mismos que se consideran dentro de la planificación territorial.

Algunos autores como Sandoval (2014), Correira de Andrade (1996) y Geiger (1996) coinciden que en la actualidad la planificación territorial, hace referencia a la planificación dentro territorio. Es por esto que la planificación es muy importante dentro de un territorio, por la relación que tiene desde su creación ya que mantenían un pensamiento de gestión vinculado al poder público dentro del espacio determinado.

Para Quiñonez et al. (2013), la planificación territorial tiene como objetivo principal analizar la problemática existente generados por las acciones del ser humano reflejándose en desigualdades en varios escenarios como son: ambientales, económicas, culturales y/o sociales, de los diversos habitantes. Mientras tanto Sandoval (2014), indica que dentro de planificación territorial es importante y necesario asociar elementos importantes como: la planificación, la infraestructura, localización de actividades, zonificación entre otros.

Po lo tanto se deduce que la planificación territorial, se encuentra vinculada a diversas políticas que pueden ser locales, regionales, nacionales y comunitarias entre ellas especialmente las de desarrollo territorial, urbanismo y vivienda, así como de infraestructura. En la practica la planificación territorial se asocia con las disciplinas del ordenamiento territorial, con un solo fin de dar solución a los desequilibrios existentes en el territorio ocasionados por la intervención del ser humano.

El autor Massiris (2008), indica que en la actualidad la planificación territorial, "se plantea desde una mirada multisectorial y pluridisciplinaria, tendiente a lograr un desarrollo integral. Los países El Salvador, Costa Rica, México, Brasil, Uruguay y 
Colombia han impulsado un desarrollo regional equilibrado y justo incluyendo a las sociedades en la participación y toma de decisiones dentro de cada territorio".

El mismo autor, hace un recorrido conceptual y de las prácticas acerca del Ordenamiento Territorial llevadas a cabo en 15 países latinoamericanos ha identificado que el ordenamiento territorial se ha generado de manera distinta ya que se encuentran vinculados a las políticas urbanísticas, ambientales, referentes a desarrollo regional y descentralización. La justificación del replanteamiento de desarrollo de los 15 países estudiados "se fundamenta en cinco razones: a) La profundización de las brechas entre ricos y pobres. b) La incapacidad de las fuerzas del mercado para impulsar un desarrollo territorial o regional equilibrado y justo. c) La demanda creciente de la sociedad por una mayor participación en la gestión del desarrollo. d) El propósito de alcanzar la sostenibilidad ambiental del desarrollo para garantizar la disponibilidad futura de recursos naturales. e) La necesidad de reducir los riesgos de catástrofes por fenómenos naturales o sociales" (Massiris, 2008, p. 4).

Para complementar el acápite anterior Masiris (2008), indica que el modelo de desarrollo en el Ecuador mantiene procesos en donde se buscan alternativas para proporcionar solución a "los diversos problemas de desigualdad e iniquidad social y territorial y de aprovechamiento insostenible de los recursos naturales que el modelo económico neoliberal no solo no ha podido resolver, sino que en la mayoría de los casos ha profundizado".

\section{Aspectos teóricos y enfoques del Ordenamiento territorial}

Con respecto al ordenamiento territorial los autores Arthur et al, (2001) manifiestan que se trata de unificar la planificación biofísica con la socioeconómica. Mientras que los autores Leingthton (1998) y Massiris (2005), comparten que la ordenación territorio tiene que ser autoritaria, coordinada e incluyente de políticas públicas sectoriales para poder generar progreso a largo plazo de los aspectos ambientales, económicos, culturales y sociales, con un fin de generar un equilibrio territorial.

Por otro lado, Estaba (1999), expresa que el ordenamiento territorial se refiere a acciones del Estado con el propósito de identificar una organización del uso de la tierra, basándose en la distribución de las oportunidades de expansión, considerando las necesidades y potencialidades. Siguiendo a Baeriswyl (2001) y Gómez (2007), coinciden que el "Ordenamiento Territorial es un proceso, en el cual no sólo existe preocupación por el uso sustentable de los recursos naturales, sino que incluye aspectos institucionales, políticos, socio-culturales y económicos".

Entonces la ordenación territorial tiene como objeto el desarrollo que se genera en el territorio mediante políticas públicas sectoriales que considera los siguientes factores: económicos, sociales, culturales, ambientales y políticos e institucionales. Dando 
cumplimiento a los objetivos propuestos en la Carta Europea del Territorio 1983 que son: “a) El desarrollo socioeconómico equilibrado de las regiones, b) La mejora de la calidad de vida; c) La gestión responsable de los recursos naturales y la protección del medio ambiente; d) La utilización racional del territorio". También se plasman estos objetivos en la mayoría de leyes de ordenación del territorio.

Según Castro indica que "la vigencia del plan de ordenamiento territorial se basa en tres componentes: 1) Estructural y de contenidos urbanos, que tendrá una vigencia en el largo plazo de 3 periodos de administraciones municipales y la revisión que se haga a ese POT deben enmarcarse en el inicio de un nuevo periodo de gobierno. 2) El contenido de mediano plazo, el cual tendrá una duración mínima de dos periodos gubernamentales de alcaldía, 3) El largo plazo, que quiere decir que los programas de ejecución estarán dentro de un periodo de gobierno municipal como mínimo". (2016, p.15).

\section{Aspectos teóricos sobre la planificación territorial y la distribución equitativa de beneficios en el Ecuador.}

Luego de este análisis realizado en los acápites anteriores se puede entender que existe una relación entre la planificación territorial y el ordenamiento territorial, siendo un proceso integral en donde incluyen los criterios de gestión administrativa y financiera que deben ser articulados a la planificación mediante gestión.

Gómez (2003), manifiesta que ordenar el territorio es "identificar, distribuir organizar y regular las actividades que realiza el ser humano en un determinado territorio"; es decir a ordenación de los usos del suelo. También complementa Gómez (2003), que la ordenación territorial al ser muy compleja se debe considerar las siguientes palabras como: promoción, ordenación, regulación, funcionalidad, integración y equilibrio, las mismas caracterizan a la ordenación territorial y a sus objetivos planteados, así mismo su importancia varía según el territorio a ser estudiado.

Cabrera (2013), expresa a inicio de los años noventa inicia en el Ecuador la elaboración de los Planes de Ordenamiento Territorial (PDOT) y las relaciones jurídicas, también reconocían la transversalidad e intersectorialidad de la ordenación del territorio que incluían elementos urbano-regional y de la planificación ambiental, sin embargo, no solventaban los grandes problemas de los planes ambientales, socioeconómicas y urbanísticos, esto generaba problemas debido a las normas que contenía cada PDOT.

\section{Marco Normativo}

Así mismo dentro del marco normativo contamos con las Constitución 2008 el título V, en los Art. 263 a 267, establece las diversas competencias exclusivas para cada uno de los niveles de gobierno, una de las principales competencias del GAD parroquial es: "Planificar el desarrollo y formular los correspondientes planes de ordenamiento 
territorial, de manera articulada con la planificación nacional, regional, provincial, cantonal y parroquial," en la actualidad se encuentra normada por el Código Orgánico de Organización Territorial, Autonomía y Descentralización (COOTAD), el Código Orgánico de Planificación y Finanzas Publicas (COPFP), la Ley Orgánica de Organización Territorial, Uso y Gestión de Suelo (LOOTUGS).

El Art. 13 del COPFP, determina que "La planificación de desarrollo y ordenamiento territorial es competencia de los gobiernos autónomos descentralizados en el marco de sus competencias. Se ejercerá a través de sus planes y demás instrumentos, en articulación y coordinación entre niveles de gobierno, en el ámbito del Sistema Nacional Descentralizado de Planificación Participativa."

El Art. 276 de la Constitución de la Republica 2008, establece que el régimen de desarrollo contiene siete objetivos siendo el principal el objetivo 6. "Promover un ordenamiento territorial equilibrado y equitativo que integres y articule las actividades socioculturales, administrativas, económicas y de gestión, y que coadyuve a la unidad del Estado".

El Art. 9 de la LOOTUGS define: "El ordenamiento territorial es el proceso y resultado de organizar espacial y funcionalmente las actividades y recursos en el territorio, para viabilizar la aplicación y concreción de políticas públicas democráticas y participativas y facilitar el logro de los objetivos de desarrollo. La planificación del ordenamiento territorial constará en el plan de desarrollo y ordenamiento territorial de los Gobiernos Autónomos Descentralizados. La planificación para el ordenamiento territorial es obligatoria para todos los niveles de gobierno.”

En el Reglamento de la LOOTUGS el Art. 8 establece que "se debe actualizar los Planes de Desarrollo y Ordenamiento Territorial obligatoriamente en las siguientes circunstancias: a) Al inicio del periodo de gestión de autoridades locales, b) cuando un proyecto nacional de carácter estratégico se proponga en la jurisdicción territorial y debe adecuar su PDOT a los lineamientos derivados de la respectiva planificación especial, c) Por fuerza mayor, como la ocurrencia de un desastre".

El Art. 41 del COPFP, establece que "los planes de desarrollo son las directrices principales de los GADs respecto a las decisiones estratégicas de desarrollo en el territorio, el mismo tendrá una visión a largo plazo y serán materializados a través del ejercicio de sus competencias". Dentro del Art. 43 del mismo código indica que los planes de Ordenamiento Territorial es la herramienta primordial para la planificación del territorio, con el fin de generar desarrollo dentro de los componentes de asentamientos humanos, económico productivo y biofísico considerando las potencialidades del territorio, guiándose en los lineamientos planteados para alcanzar un modelo territorial de largo plazo, establecido por el nivel de gobierno correspondiente. 
El Art. 42 del COPFP determina que en concordancia a las con las disposiciones del COOTAD, los planes de desarrollo y ordenamiento territorial de los gobiernos autónomos descentralizados deberán contener al menos lo siguiente: “1). Diagnóstico, 2). Propuesta y 3). Modelo de Gestión".

Por otra parte, Zamora y Carrión (20013), analizan e indican que los PDOT, son instrumentos de planificación, mismos que deberán ser aplicados a un territorio determinado para generar desarrollo equilibrado, es por esta razón que este instrumento debe ser: Participativo, Coherentes, Prospectivos, Flexibles, Operativos, Estratégicos.

La actual guía de para formulación/ actualización del (PDOT) Parroquial 2019, indica que el propósito del PDOT es materializar la visión territorial en un tiempo determinado que puede ser: en corto (máximo a 2 años), mediano (máximo a 5 años), y largo plazo (máximo a 20años), "con las directrices e intervenciones concretas respecto del desarrollo económico, la conservación, el uso y gestión de los recursos naturales, el patrimonio cultural; la prevención y reducción de riesgos; la gestión del cambio climático; los sistemas de redes de servicios, transporte, movilidad, accesibilidad, energía y telecomunicaciones; rol de sus asentamientos humanos, tanto en el ámbito urbano como rural. Para ello se debe considerar: a) El plan de trabajo de las autoridades electas; b) Las competencias exclusivas del GAD; c) Generar estrategias de articulación con otros niveles de gobierno y d) Disponibilidad de los recursos económicos".

En la amplia bibliografía revisada se puede indicar que el proceso del ordenamiento de un territorio permite identificar las diversas aspiraciones y necesidades de los sectores más desprotegidos los mismos deben esta plasmados en los PDOT para plantear proyectos que ayuden a un desarrollo equilibrado del territorio. La creación y ejecución de proyectos es la parte fundamental para que todo este proceso se pueda ir a los diferentes cambios que se dan en el ámbito social, económico, productivo, ambiental y político.

\section{Distribución de los recursos del estado central a las localidades}

Para Quiroga et al., (2019), indica que la distribución de los beneficios debe tener como finalidad un desarrollo en el ámbito social y económico, mismos que tienen una finalidad de mejorar la calidad de vida de la población.

Mientras que en el Art. 270 de la Constitución de la República del Ecuador 2008 en relación a los recursos financieros los GADs, establece que "Los gobiernos autónomos descentralizados generarán sus propios recursos financieros y participarán de las rentas del Estado, de conformidad con los principios de subsidiaridad, solidaridad y equidad".

Según el Art 297 de la Constitución de la Republica del Ecuador 2008 indica que "los presupuestos de los gobiernos autónomos descentralizados y sus empresas públicas se sujetarán a sus propios planes, en el marco del Plan Nacional de Desarrollo y sin 
menoscabo de sus competencias y autonomías. El Plan Nacional de Desarrollo articula el ejercicio de las competencias de cada nivel de gobierno". También se debe considerar que las metas establecidas en el PDOT en este caso parroquial deberán ayudar al cumplimiento de las metas propuestas en el Plan Nacional de Desarrollo.

En el Art. 49 del COPFP, establece que "Los planes de desarrollo y de ordenamiento territorial serán referentes obligatorios para la elaboración de planes de inversión, presupuestos y demás instrumentos de gestión de cada gobierno autónomo descentralizado".

Para finalizar en el Art. 49 del COPFP, indica que se debe realizar un seguimiento y evaluación de las metas establecidas dentro de los Planes de Ordenamiento Territorial y de esta manera poder determinar el cumplimiento o incumplimiento de este instrumento de planificación.

Por ende, los Gobiernos Autónomos Descentralizados deben regirse a las leyes antes expuestas para la distribución del presupuesto. De tal manera que faciliten la redistribución de la riqueza y disminución de inequidades, observando principios como la solidaridad, oportunidad y equidad territorial.

\section{Presupuesto del GAD Parroquial}

Según el Art. 293 de la Constitución de la Republica del Ecuador (2008), indica que "La formulación y la ejecución del Presupuesto General del Estado se sujetarán al Plan Nacional de Desarrollo. Los presupuestos de los gobiernos autónomos descentralizados y los de otras entidades públicas se ajustarán a los planes regionales, provinciales, cantonales y parroquiales, respectivamente, en el marco del Plan Nacional de Desarrollo, sin menoscabo de sus competencias y su autonomía".

Para Orellana y Salinas., (2011) y salinas, indican "los objetivos del sistema de presupuesto que son los siguientes:

- Orientar los recursos disponibles para el logro de los objetivos.

- Constituirse en la expresión de los proyectos y programas para el periodo determinado.

- Asegurarse que las etapas del ciclo presupuestario se cumplan antes de iniciarse el periodo.

- Que la etapa de aprobación del presupuesto se cumpla antes de iniciarse el periodo.

- Asegurar que la ejecución presupuestaria se desarrolle de acuerdo a las necesidades del programa y proyecto en cuestión.

- Facilitar el control interno. 
- Utilizar la ejecución y evaluación para corregir desviaciones en la programación de acciones.

- Utilizar el presupuesto como sistema de planificación y herramienta de administración.

- Realizar y presentar información comparativa entre lo presupuestado y lo ejecutado.

- La distribución del prepuesto es importante porque brinda los siguientes beneficios:

- Redistribución del ingreso

- Equidad tributaria

- Definir fuentes de financiación

- Combatir la pobreza

- Coadyuvar en la competitividad

- Cumplir metas del plan de desarrollo y programa de gobierno

- Satisfacer necesidades básicas de la población"

De acuerdo a la información analizada se puede indicar que el estado y los GADs tiene como función principal de realizar una redistribución de los ingresos y la erradicación de la pobreza, mediante la elaboración de los programas y proyectos que se justifiquen en los PDOT para que se pueda realizar el gasto.

\section{Metodología}

Para efecto de la presente investigación se toma como base fundamental investigar la distribución de beneficios dentro de la parroquia Chumblín del cantón San Fernando, principalmente tomando en cuenta la distribución de los beneficios dentro del territorio. En este trabajo de investigación se plantea el método científico, como el procedimiento para vincular a la investigación con la realidad en producción del conocimiento.

El método científico minimizara la influencia de la subjetividad en este trabajo de investigación empleando instrumentos investigativos para acceder al conocimiento, en este caso se optó por las entrevistas diseñadas para que puedan ser utilizadas de acuerdo al escenario planteado, que se han presentado en el periodo 2009-2019 sobre la distribución de beneficios en la parroquia Chumblín.

Esta investigación por sus objetivos específicos planteados está clasificada como una investigación de campo y escritorio, por cuanto se realizó en la parroquia Chumblín.

\section{Metodología e instrumentos para recopilación de datos}

Las metodologías empleadas en este trabajo de investigación son: 


\section{Fuentes primarias}

Se utilizará esta metodología de recolección de información, para profundizar en la investigación del problema ¿Cómo lograr una distribución adecuada de los beneficios de la planificación territorial de la parroquia Chumblín, cantón San Fernando?

\section{Entrevista}

Este instrumento de recolección de información servirá de ayuda para verificar la veracidad del problema, identificado es ¿Cómo lograr una distribución adecuada de los beneficios de la planificación territorial de la parroquia Chumblín, cantón San Fernando?

Elemento: Presidentes y actores sociales del centro parroquial y sus recintos.

- Etapa 1: Se visitará a los presidentes y actores sociales de los recintos de la parroquia Chumblín

- Etapa 2: Se hará conocer el objetivo de la entrevista

- Etapa 3: Se procederá a realizar las interrogantes planteadas.

- Alcance: Parroquial Chumblín

- Periodo: 2009-2019

La entrevista servirá para la recolección de información relevante, confiable y oportuna en el desarrollo del trabajo de investigación; se entrevistará a los presidentes y actores sociales del centro parroquial y recintos de la parroquia.

\section{Instrumento de la investigación}

El instrumento que utilizará será el cuestionario, indispensable para la recolección de información, útil para profundizar el problema y tema de estudio, resultados que definirán la identificación de su posible solución.

En el diseño del instrumento se considerarán las diferentes actividades con sus respectivos fases y actividades; tal como lo indica el siguiente cuadro:

\begin{tabular}{|c|c|}
\hline FASES & ACTIVIDADES \\
\hline \multirow{6}{*}{$\begin{array}{l}\text { DESCRIPCIÓN DE LOS } \\
\text { OBJETIVOS Y DEL } \\
\text { CUESTIONARIO }\end{array}$} & Exploración y análisis del problema y tema a ser investigado. \\
\hline & . $\quad$ Explicación del propósito del cuestionario. \\
\hline & $\begin{array}{l}\text { Revisión de variada bibliografía y trabajos relacionados con la } \\
\text { construcción del cuestionario. }\end{array}$ \\
\hline & . $\quad$ Consulta a expertos sobre construcción del cuestionario. \\
\hline & Identificación de las personas a ser entrevistadas. \\
\hline & Determinación de los objetivos, contenidos del cuestionario. \\
\hline \multirow{3}{*}{$\begin{array}{l}\text { DISEÑO DEL } \\
\text { CUESTIONARIO }\end{array}$} & Estructuración de las preguntas \\
\hline & Estructuración del cuestionario \\
\hline & Elaboración del cuestionario \\
\hline \multirow{4}{*}{$\begin{array}{l}\text { PRUEBA PILOTO DEL } \\
\text { CUESTIONARIO }\end{array}$} & Revisión por expertos del cuestionario planteado. \\
\hline & $\begin{array}{l}\text { Fortalecimiento de la redacción del instrumento de acuerdo a } \\
\text { recomendaciones de los expertos }\end{array}$ \\
\hline & . $\quad$ Aplicación del cuestionario una muestra piloto. \\
\hline & Análisis de resultados. \\
\hline
\end{tabular}


CUESTIONARIO · Impresión del instrumento DEFINITIVO

Cuadro 1: Fases ya actividades para la elaboración del cuestionario

Fuente: Elaboración propia.

Por las fuentes de consulta es bibliográfica, por cuanto se fundamenta en libros, folletos y demás referentes a los temas de ordenación territorial y distribución de los beneficios.

\section{Fuentes secundarias}

Se utilizará esta metodología de recolección de información, de los PDOTs, POA, PACs y registros de los proyectos al sistema del SIGAD de la parroquia Chumblín en el periodo 2009-2019, para profundizar en la investigación del problema sobre la distribución de los beneficios de la planificación territorial.

\section{Resultados}

En el presente acápite, se detallan los resultados de las entrevistas, mismos que expresa la perspectiva de los presidentes y grupos focales de los siguientes recintos: el Cisne, Bellavista, Nube de Cruz Pamba y el Centro Parroquial de la parroquia Chumblín, con respecto a la distribución de los beneficios generados en el periodo 2009-2019.

Planificación. - Con relación a la planificación dentro de la parroquia Chumblín, se pudo evidenciar que el $52 \%$ de los entrevistados no tienen conocimiento sobre la planificación territorial, así mismo es preocupante que el $57 \%$ de los entrevistados no han sido participes de los procesos de desarrollo y ordenamiento territorial, siendo los mismos representantes de cada uno de los recintos, sin embargo el $57 \%$ indica que en el periodo 2009-2019 si se ha generado una inversión planificada pero no de forma equitativa ya los beneficios eran para ciertos grupos de recintos.

También es alarmante conocer que el $70 \%$ de los entrevistados expresan que para la elaboración y ejecución de los proyectos no se han considerado las necesidades primordiales de la población. Sin embargo, de las personas entrevistadas el 65\% indican que la empresa INV Minerales tiene más influencia en la Planificación Territorial debido a los proyectos que se generan con facilidad de los diversos componentes que se establecen en el PDOT, así mismo el 22\% indican que el GAD Parroquial tiene más influencia dentro del territorio; siendo menos perceptibles el GAD Municipal, el GAD Provincial entre otros.

A pesar que no ha existido un empoderamiento de la población en los procesos de planificación, dentro de la parroquia Chumblín ha sido evidente que ha existido un desarrollo en el periodo 2009-2019, esto lo considera el 57\% de los entrevistados indican que existió desarrollo en el ámbito Social, mientras que el 30\% indica que ha existido desarrollo en el ámbito productivo y el $13 \%$ en ámbito ambiental. A pesar de existir una inequidad en la distribución de los beneficios ya que rara vez se considera las necesidades de la población para la elaboración y ejecución de los proyectos; el 59\% de los 
entrevistados indican que la ejecución de los proyectos realizados desde el año 2009-2019 han sido buenos.

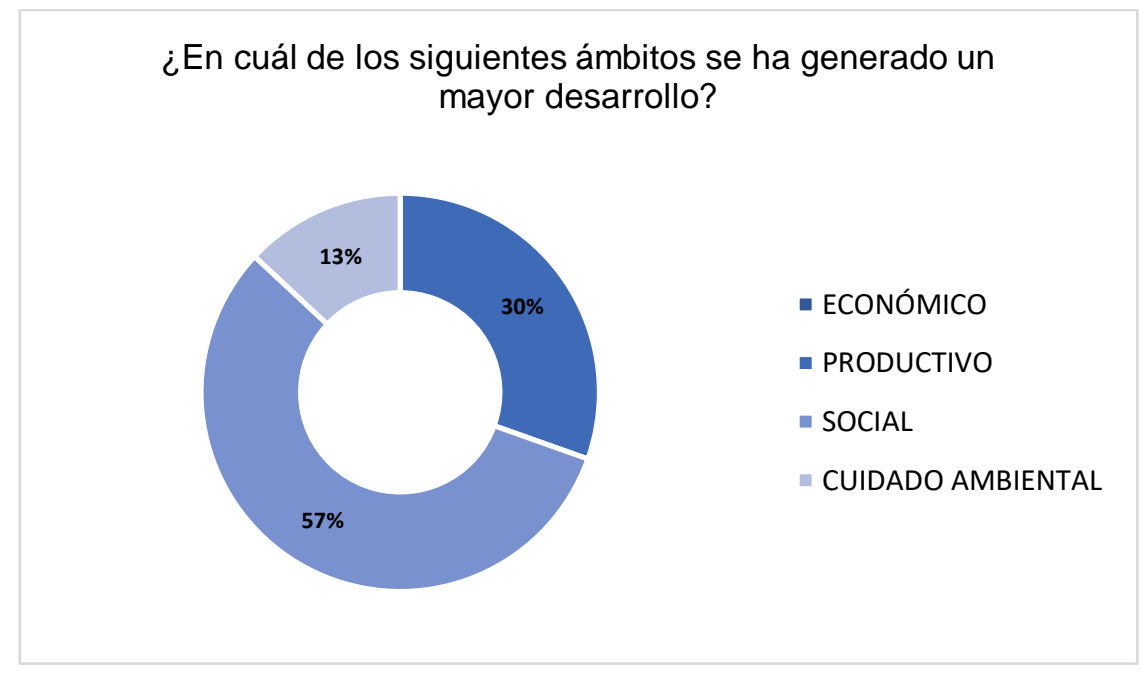

Gráfico 1: Ámbitos que ha tenido mayor desarrollo en el territorio

Fuente: Elaboración propia.

Inversión. -Con respecto al tema de inversión es preocupante ya que el $87 \%$ de los entrevistados indican que rara vez se han socializado los proyectos que se realizaron en la parroquia en el periodo 2009-2019. Dando como resultado que de las personas entrevistadas que son dirigentes de los recintos y actores sociales el $87 \%$ no tengan conocimiento sobre los presupuestos que maneja anualmente el GAD Parroquia y el $61 \%$ no tiene conocimiento del dinero que maneja la empresa INV Minerales.

Al tener conocimiento que un gran porcentaje de las personas entrevistadas indican que no conocen del presupuesto que maneja el GAD Parroquial, existe una contradicción ya que el $87 \%$ coinciden que el GAD Parroquial es quien realiza mayor inversión en los proyectos realizados en el territorio.

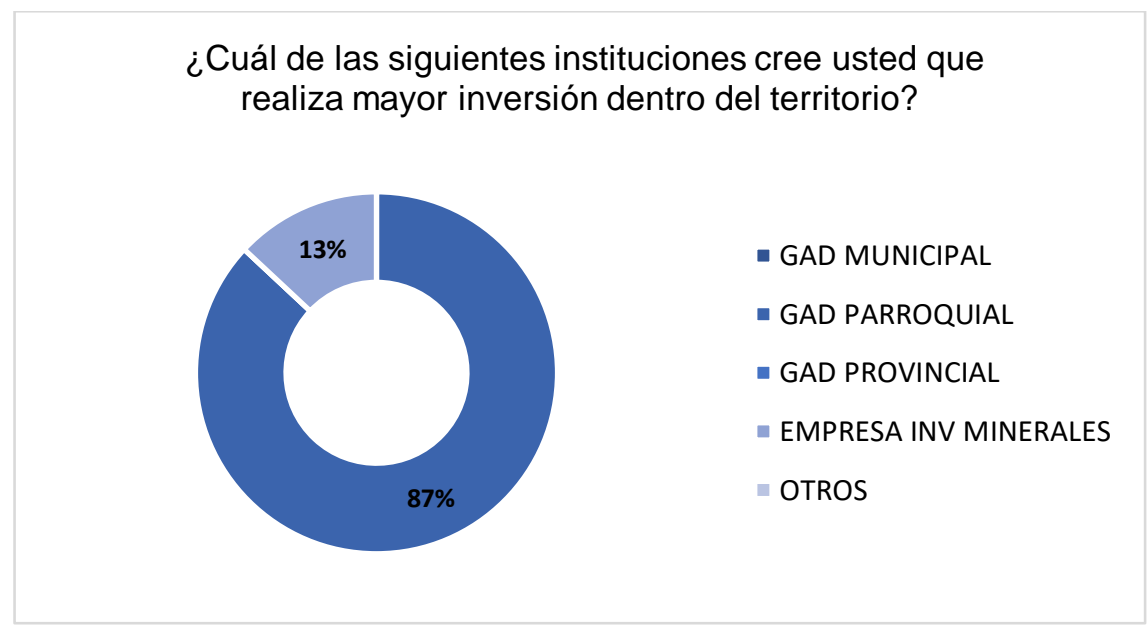

Gráfico 2: Instituciones que realizan mayor inversión en el territorio Fuente: Elaboración propia.

Distribución. - En cuanto al tema de distribución, el 57\% de los entrevistados indican que se realiza una mayor inversión en proyectos referentes a vialidad, mientras que el 
$17 \%$ indica que se realiza mayor inversión en los proyectos referentes a infraestructura y con el mismo porcentaje del 13\% indican que existe una mayor inversión en el ámbito productivo y social; en cuanto a proyectos para el ámbito institucional no se ha realizado inversión alguna. Se puede verificar que la inversión para los proyectos no se realiza de forma equitativa ya que se evidencia que existe mayor inversión en proyectos del ámbito vial e infraestructura.

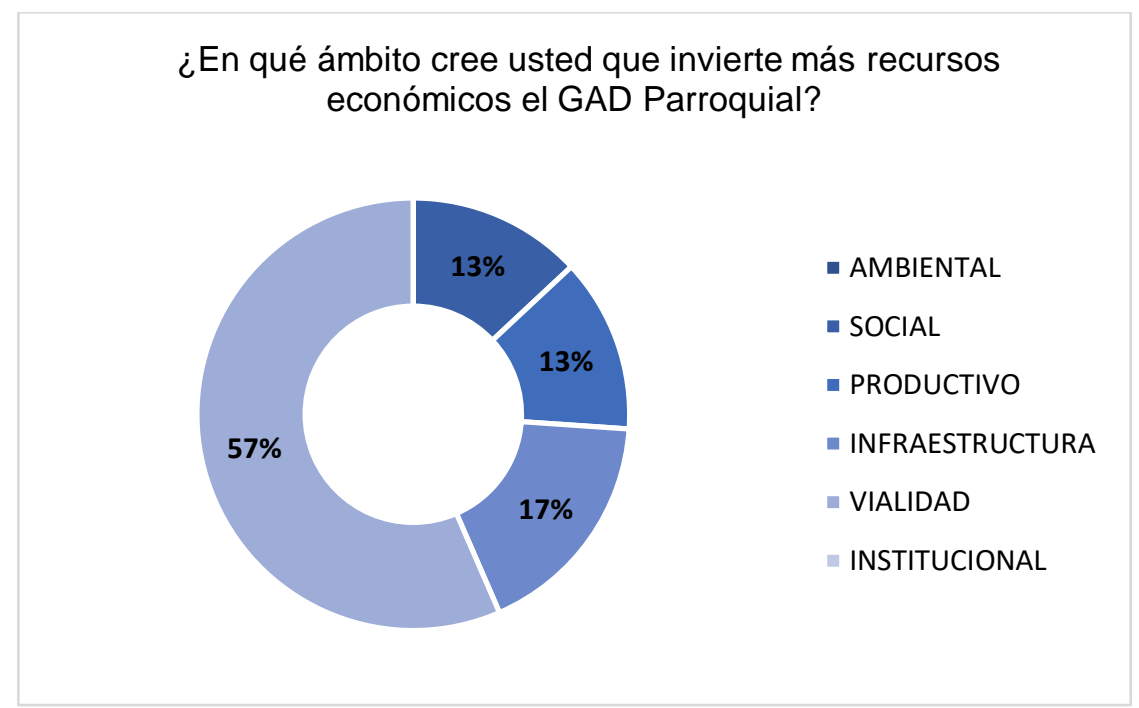

Gráfico 3: Inversión del GAD Parroquial

Fuente: Elaboración propia.

De la misma manera las personas entrevistadas el $31 \%$ han identificado que la empresa INV Minerales invierte más recursos económicos en el ámbito de producción, mientras que en igual porcentaje del $26 \%$ invierte en el ámbito social e infraestructura, el $17 \%$ existe inversión en el ámbito de vialidad, mientras tanto en el ámbito ambiental e institucional no identifican que se realice mayor inversión. Es por ello que se pudo constatar que no se distribuye de forma equitativa los recursos económicos que invierte la empresa INV Minerales ya que solo realiza mayor inversión en ciertos ámbitos.

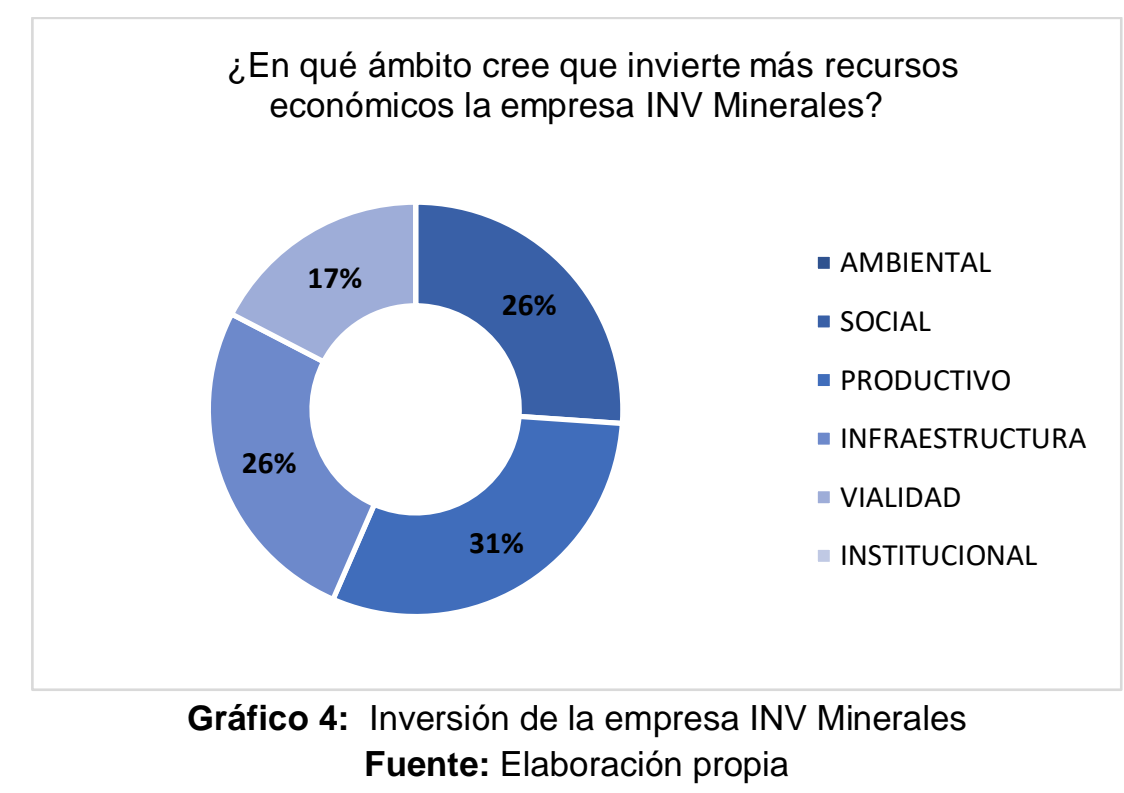


De las personas entrevistadas el 65\% indica que la inversión que se realiza en el territorio lo realizan de manera injusta ya que de los proyectos generados en la mayoría no se consideran las necesidades de la población, provocando que sean beneficiarios ciertos grupos focales de los recintos más desarrollados. Así mismo de personas entrevistas el $52 \%$ indican que no han recibido ningún beneficio de los proyectos realizados en el territorio, igualmente el $57 \%$ indica que el recinto más beneficiado es el recinto el cisne y el $39 \%$ indica que el centro parroquial es el más beneficiado, resultando que el recinto el Cisne y el centro Parroquial son los que más proyectos han recibido en el periodo 20092019.

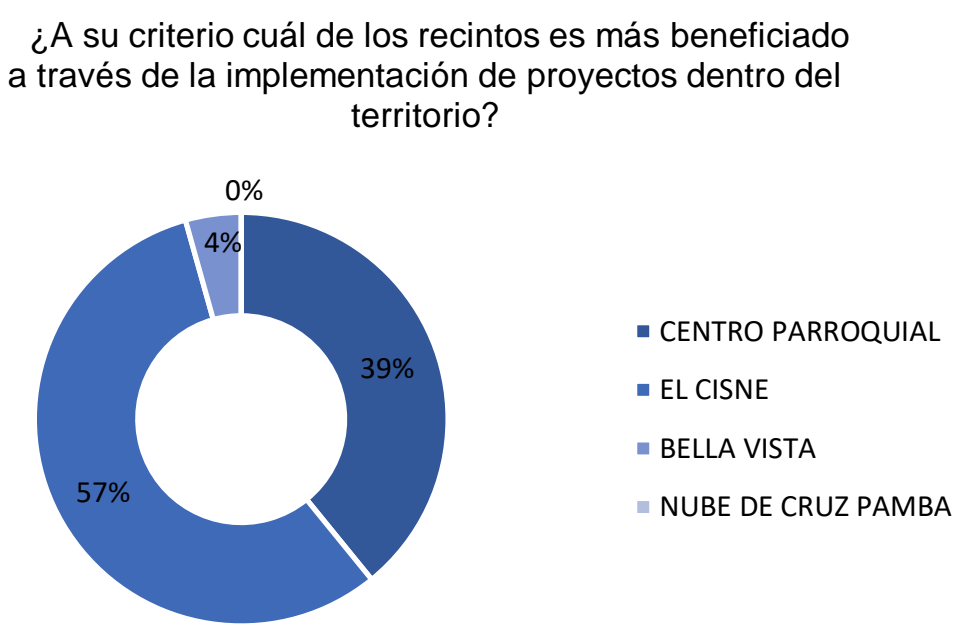

Gráfico 5: Recintos más beneficiados a través de proyectos Fuente: Elaboración propia

\section{Análisis de la información secundaria:}

De acuerdo a la recolección de información secundaria se procedió con la revisión de los Planes de Desarrollo y Ordenamiento Territorial, Planes Operativos Anuales y los registros subidos al sistema del SIGAD del periodo de análisis 2009-2019, en los mismos se pudo identificar el número y el valor de inversión de los proyectos realizados de los componentes: Biofísico, Sociocultural, Económico Productivo, Asentamientos Humanos, Movilidad y Conectividad y Político Institucional; obteniendo como resultado que se han generado 8 proyectos tanto en el componente Económico Productivo como en el componente Movilidad y Conectividad, resultando estos componentes con más proyectos realizados, por otro lado el componente de Asentamientos Humanos a generado 7 proyectos y el componente Biofísico y Social ha generado 5 proyectos cada uno de ellos y dentro del componentes Político Institucional se ha generado un solo proyecto en todo el periodo de estudio, se pudo verificar que el en el componente político institucional no era considerado desde el 2009-2014 dentro de la planificación, siendo nula la participación de la ciudadanía en estos procesos de planificación. 
Sin embargo, en el siguiente grafico se puede observar que el GAD Parroquial ha realizado mayor inversión económica en el componente Movilidad y Conectividad y Asentamientos Humanos, mientras que el componente Político Institucional es el que menos recursos se ha invertido durante el periodo de análisis ya que el mismo fue considerado en la planificación del 2014-2019, siendo esta la razón por la cual la gente desconocía de los procesos y los proyectos realizados dentro de la planificación del territorio.

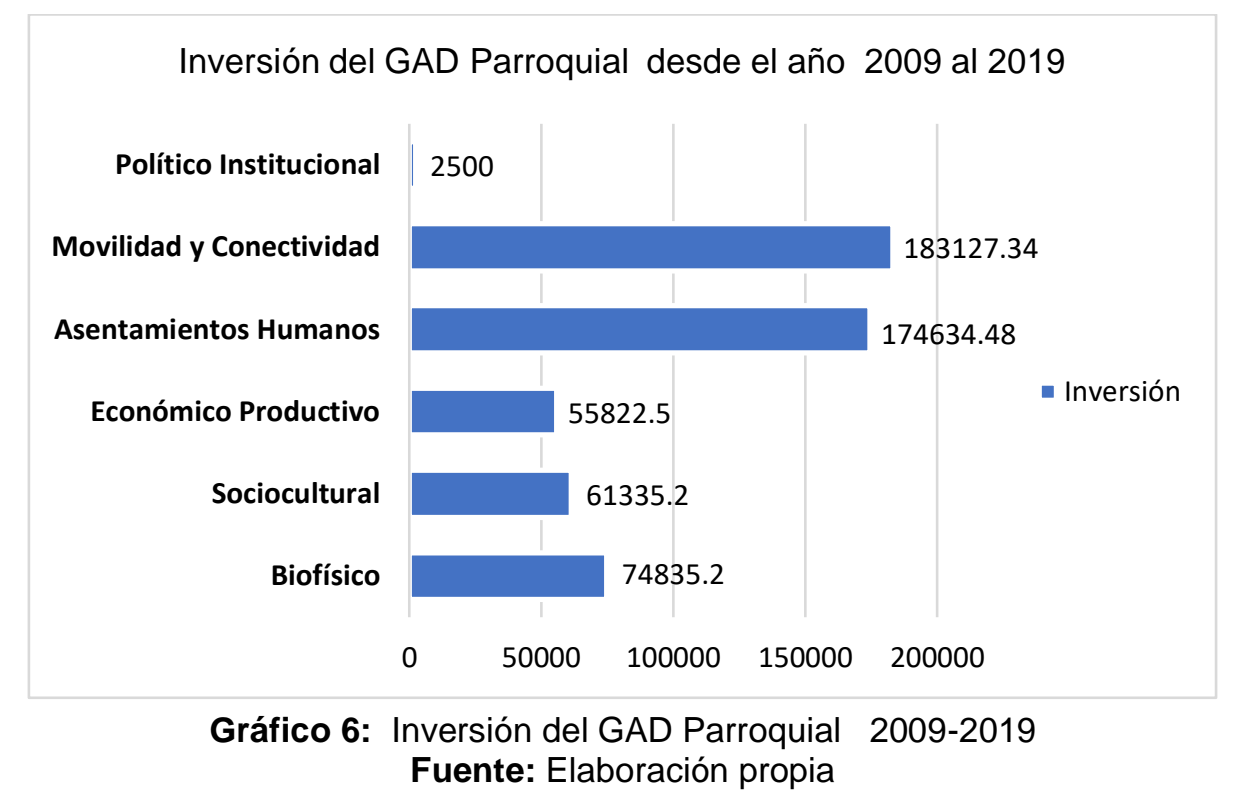

Dentro de esta investigación se ha considerado importante en realizar el análisis sobre la inversión que realiza la empresa privada INV Minerales dentro del territorio ya que de las personas entrevistadas el $65 \%$ considera que la misma tiene mayor influencia en la planificación del territorio, cabe indicar en cada administración se firma un convenio de cooperación interinstitucional en el mismo se detalla de qué manera se invertirá los recursos en los proyectos que proponga el GAD Parroquial, también detalla que para la entrega de los recursos económicos se basaran en cinco ejes que son: Gestión y servicios comunitarios, Producción, Educación y Cultura; Fomento del talento (capacitaciones) y Salud, Seguridad y Ambiente; los mismos que aportaran a cada uno de los componentes establecidos dentro del PDOT parroquial.

En la siguiente gráfico se detalla la inversión que ha realizado que la empresa INV Minerales en el periodo 20009-2019, la misma que sigue incrementando los recursos para los componentes: Movilidad y Conectividad, Asentamientos Humanos y Económico Productivo, también cabe mencionar que dentro de los ejes propuestos por la empresa se pueden realizar inversión en Educación y Salud, siendo una ventaja para la parroquia ya que el GAD parroquial no puede realizar inversión en los mismos porque no es competencia del GAD parroquial. 
Inversión de la empresa INV Minerales desde el año 2009 al 2019

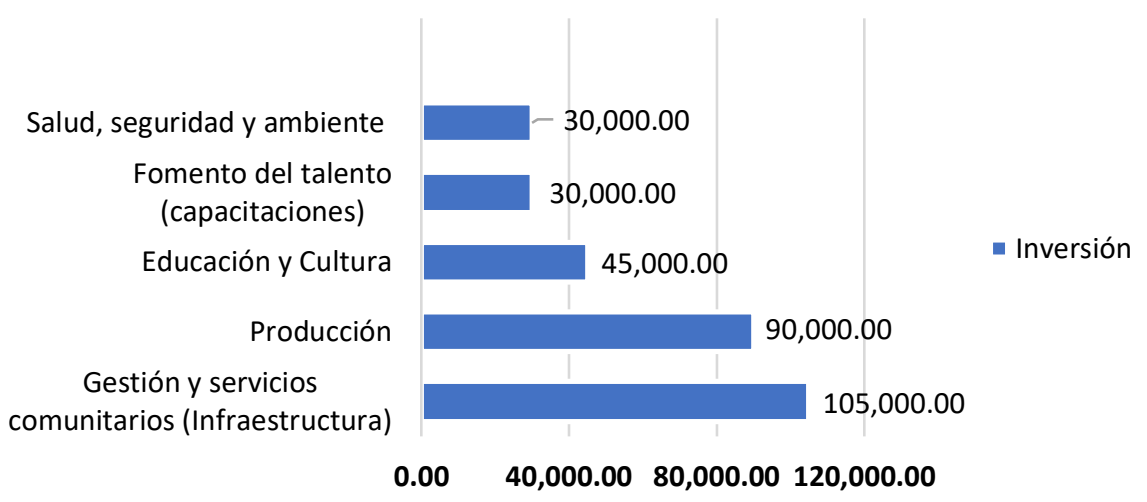

Gráfico 7: Inversión de la empresa INV Minerales en el periodo 2009-2019

Fuente: Elaboración propia

Para un mayor entendimiento en la siguiente grafica se ha realizado un análisis global de los recursos unificados tanto del GAD Parroquia y la empresa INV Minerales, en donde se sigue verificando que los recursos no se encuentran distribuidos de manera equitativa, debido a que se sigue aportando en mayor porcentaje a los componentes Movilidad y Conectividad, Asentamientos Humanos y Económico Productivo, mientras que el componente Biofísico y Político Institucional son los que menos recursos reciben.

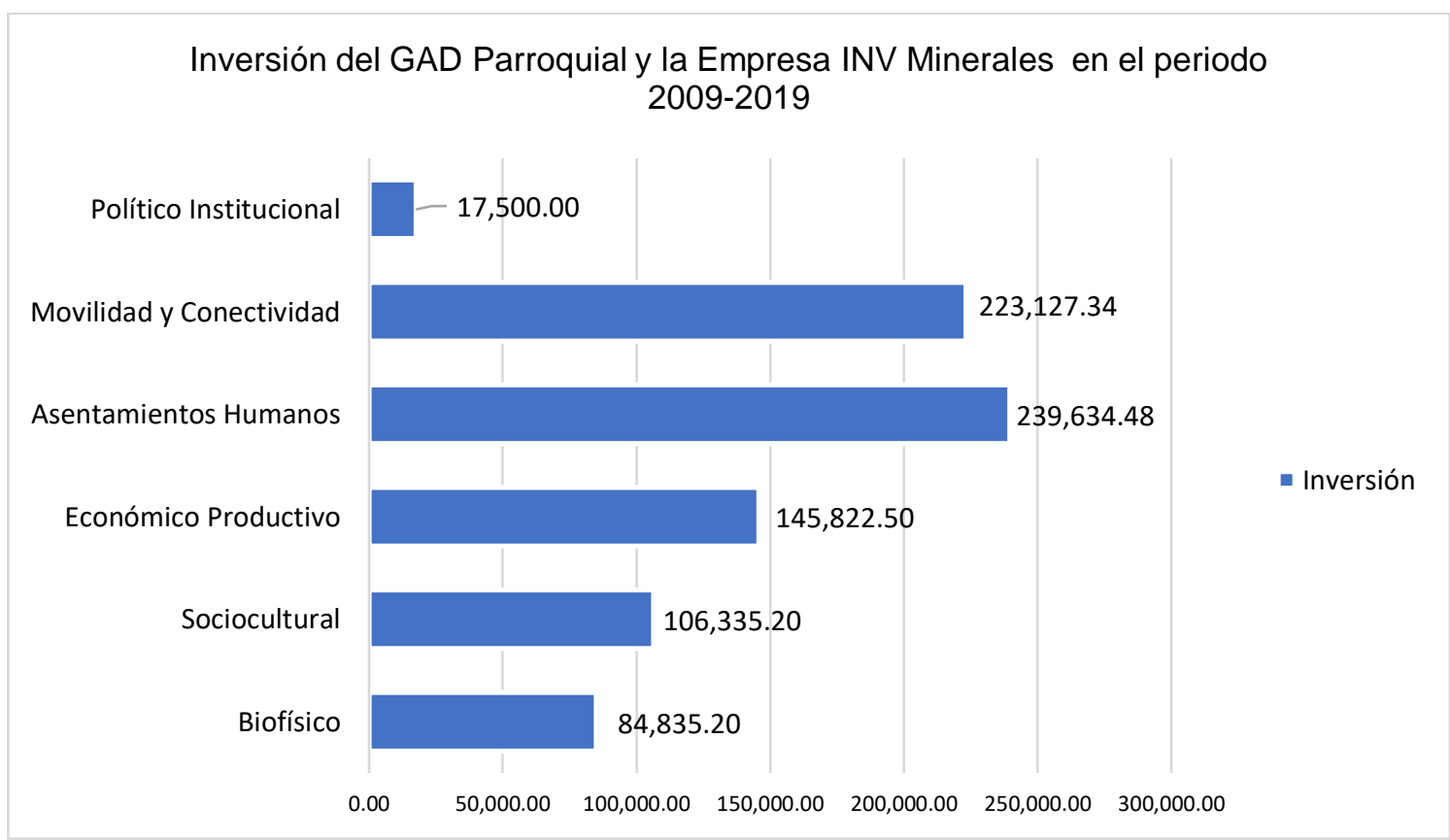

Gráfico 8: Inversión del GAD Parroquial y la empresa INV Minerales Fuente: Elaboración propia

\section{Propuesta.}

\section{Estrategias:}

- En base a la información obtenida mediante entrevistas es evidente la poca o nula participación de la población en los procesos de planificación; por lo que es 
necesario generar proyectos dentro del componente Político Institucional con el fin de generar mecanismos de participación ciudadana para una planificación efectiva, consolidada y eficaz, priorizando las necesidades de la población, con la finalidad de lleguen a ser actores principales del desarrollo de su territorio.

- Difundir y promocionar a través de medios digitales, emisoras o canales locales los procesos de planificación, ejecución de presupuestos participativos y asambleas ciudadanas realizadas por el GAD Parroquial, socializando y transparentando los actos administrativos a toda la población, a su vez, generar convenios de cooperación interinstitucional con la Prefectura del Azuay, Municipio de San Fernando o CONAGOPARE AZUAY, con el fin generar mayor inversión en el territorio y promocional los atractivos turísticos y culturales de la parroquia Chumblín.

- Coordinar y articular la implementación de proyectos en el territorio, con diversas instituciones actoras del desarrollo parroquial, ampliando la cobertura $y$ mejorando los beneficios.

- Jerarquizar los asentamientos humanos con una valoración incluyente y justa, de manera que los asentamientos menos servidos tengan un peso inversamente proporcional a aquellos mejor servidos y a su vez esto se verá reflejado en la distribución de planes, programas y proyectos para el territorio, reduciendo las brechas de desigualdad e inequidad.

- Distribuir una parte del presupuesto para una planificación equitativa y el otro porcentaje sea distribuido de acuerdo a factores de ponderación relacionados a NBI, numero de población y extensión territorial.

\section{Conclusiones}

- Se realizado un análisis de la parte de la propuesta y modelo de gestión de los PDOTs de la parroquia Chumblín desde el periodo 2009-2019, los POAS, PAC y registro de los proyectos al sistema del SIGAD, mismo que permitió identificar el número de los proyectos y montos de los proyectos del componente Biofísico, Socio Cultural Económico Productivo, Asentamientos Humanos, Energía y Conectividad y Político Institucional.

- Se ha identificado que el GAD Parroquial y la empresa INV Minerales invierte más recursos económicos en el componente Económico Productivo, Asentamientos Humanos y Energía y Conectividad, mientras que los componentes de Biofísico, socio Cultural y Político Institucional se invierte pocos recursos económicos.

- El centro parroquial y el recinto el cisne son los recintos más desarrollados ya que han ejecutado mayor cantidad de proyectos realizados por parte del GAD Parroquial y la empresa INV Minerales en el periodo de análisis 2009-2019; resultando como menos desarrollados el recinto de Bellavista y Nube de Cruz pamba. 
- Existe una baja participación de la ciudadanía en temas relacionados a la planificación del territorio, provocando que la gente no tenga conocimiento sobre la planificación que se desarrolla dentro del territorio.

- También se puede indicar que para la distribución de beneficios dentro de la planificación del territorio no se considera el Marco Legal mencionado en los acápites anteriores.

\section{Referencias bibliográficas.}

Arthur C.J. van Leeuwen. (2001). Ordenamiento Territorial: Un Proceso Participativo, Sostenible y de Democratización.

ASAMBLEA NACIONAL. (2010). Segundo Suplemento Asamblea Nacional Código Orgánico De Planificación Y Finanzas Públicas Contenido Presidencia De La República. 1-57.

Cabrera, C. A. (2013). Planificación del territorio y ordenamiento territorial.

Código Orgánico Organización Territorial Autonomía Descentralización COOTAD

COOTAD. (2010). Código Orgánico Organización Territorial Autonomía Descentralización COOTAD. Registro Oficial Suplemento 303 de 19-Oct-2010, 2, 174.

Correia de Andrade, M. (1996). Territorialidades, desterritorialidades, novas territorialidades: los limites do poder nacional, e do poder local. En: Territorio: Globalizagào e Fragmentario. Sáo Paulo, Editora Hucitec, pp 213-220

Geiger, P. (1996). Des-territorializaqiio e espacializaqiio. En: Territorio: GZobaliza@b e Fragmenta@. Siio Paulo, Editora Hucitec, pp 233-246.

Gómez Orea Domingo. (2003). La Ordenación Territorial: Carácter, Alcance Y Contenido. Universidad Politécnica de 17. https://core.ac.uk/download/pdf/55526614

Gomez, D. (1994). Ordenacion del Territorio Una aproximación desde el Medio Físico L 3-Instituto Tecnoiógico Geominero de España. 118.

Gómez, G. M. (1998). Espacio, territorio y región: conceptos básicos para un proyecto nacional. Cuadernos de Geografía: Revista Colombiana de Geografía, 7(1-2), 120134.

Gouëset, V. (1999). El territorio colombiano y sus márgenes. In Territorios (Issue 1, pp. 77-94).

Latinoamericana, R. (2001). Desarrollo local en los espacios rurales. Polis: Revista de La Universidad Bolivariana, 1(2), 0. 
Massiris Á. (2005). Fundamentos conceptuales y metodológicos del ordenamiento territorial (1a edición). Editorial Jomar Ltda.

Massiris Á. (2008). Gestión del ordenamiento territorial en América Latina. Proyección, 1(Año 2008, 4).

Orellana, N. A. \& Salinas, L. M. (2011). Análisis del presupuesto de los organismos autónomos descentralizados.- Caso: Junta parroquial “San Antonio del Cantón Limón - Indanza de la provincia de Morona Santiago. Año 2010. Universidad de Cuenca, 71

Quiroga Gómez, Z. M., de Dios Martínez, A., \& Pérez Rangel, M. (2019). Enfoque poblacional en los planes de ordenamiento territorial. Retos de La Dirección, 13(1), 69-85.

SENPLADES. (2009). Plan Nacional para el Buen Vivir 2009-2013: Construyendo un Estado Plurinacional e Intercultural.

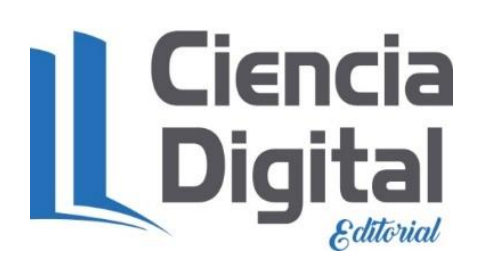




\section{PARA CITAR EL ARTÍCULO INDEXADO.}

Pazato Dutan, M. A., Flores Aguilera, D. D., \& Castillo Ortega, Y. (2021). Planificación territorial y distribución de los beneficios de la parroquia Chumblín Cantón San Fernando . ConcienciaDigital, 4(2), 74-93. https://doi.org/10.33262/concienciadigital.v4i2.1629

\section{Liencia}

El artículo que se publica es de exclusiva responsabilidad de los autores y no necesariamente reflejan el pensamiento de la Revista Conciencia Digital.

El artículo queda en propiedad de la revista y, por tanto, su publicación parcial y/o total en otro medio tiene que ser autorizado por el director de la Revista Conciencia Digital.

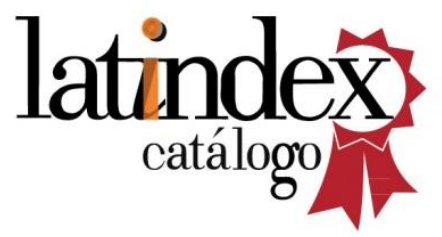

